\title{
SPECT ASSAY OF RADIOLABELED MONOCLONAL ANTIBODIES
}

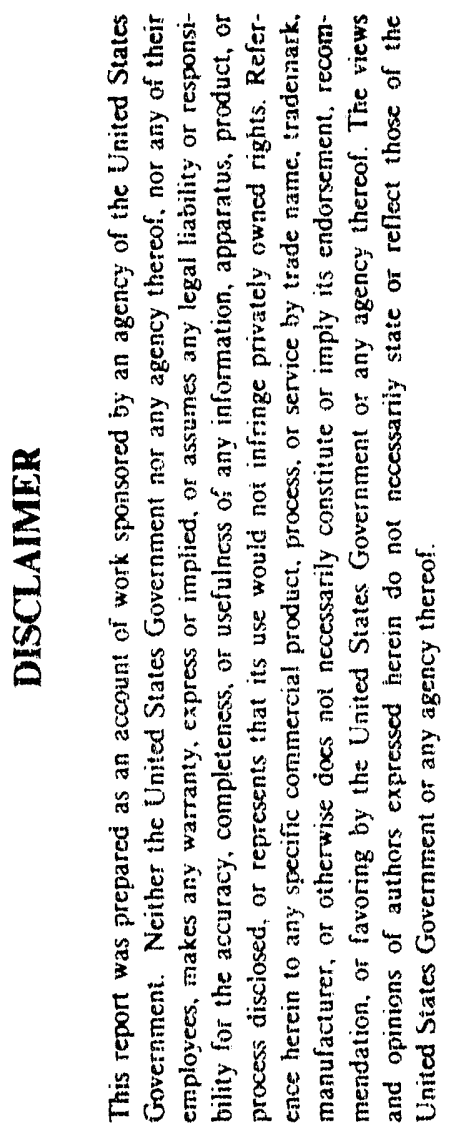

\author{
THIRD YEARLY PROGRESS REPORT \\ September 1991 - February 1992
}

\author{
Ronald J. Jaszczak, Ph.D. \\ Radiology Department \\ Duke University Medical Center \\ Durham, North Carolina 27710
}

February 1992

PREPARED FOR THE U.S. DEPARTMENT OF ENERGY

UNDER GRANT NUMBER DE-FG05-89ER60894 


\section{SPECT ASSAY OF RADIOLABELED MONOCLONAL ANTIBODIES}

\section{ABSTRACT}

The accurate determination of the bicdistribution of radiolabeled monoclonal antibodies (MoAbs) is important for calculation of dosimetry and evaluation of pharmacokinetic variables such as antibody dose and route of administration. The hypothesis of this application is that the biodistribution of radiolabeled monoclonal antibodies (MoAbs) can be quantitatively determined using single photon emission computed tomography (SPECT). Major long term objectives of this proposal are to evaluate and develop appropriate SPECT imaging approaches to quantitatively compare the in vitro and in vivo characteristics of MoAbs radiolabeled with the clinically relevant radionuclides ( ${ }^{123} \mathrm{I},{ }^{131} \mathrm{I}$, and ${ }^{111} \mathrm{In}$ ), and a nuclide with new therapeutic potential, ${ }^{211}$ At. The errors associated with the SPECT measurements will be determined with Monte Carlo simulations, by scanning phantoms containing radionuclide activities in tegions of uniform and nonuniform attenuation, and by selected in vivo studies. The ability of SPECT to quantify $123^{i}$ distributions will be assessed, and new acquisition geometries and reconstruction algorithms for improved quantification will be developed.

\section{RESEARCH EMPHASIS DURING THIRD PROJECT PERIOD}

The major thrusts during the third year have included 1) The continued development and evaluation of improved 3D SPECT acquisition and reconstruction approaches to improve quantitative imaging of radiolabeled monoclonal antibodies (MoAbs), and 2)The implementation and evaluation of algorithms to register serial SPECT image data sets, or to register 3D SPECT images with 3D image data sets acquired from positron emission tomography (PET) and magnetic resonance images (MRI). The research has involved the investigation of statistical models and iterative reconstruction algorithms that accurately account for the physical characteristics of the SPECT acquisition system. It is our belief that SPECT quantification can be improved by accurately modeling the physical processes such as attenuation, scatter, geometric collimator response, and other factors that affect the measured projection data.

\section{A. OBJECTIVES}

1) To evaluate the capability of SPECT to quantify in vitro_radioactivity distributions. The errors associated with in vitro SPECT measurements will be estimated using Monte Carlo simulations and experimental scans of phantons. SPECT measurements of radioactivity within uniformly and non-uniformly attenuating media will be evaluated.

2) To develop and evaluate methods to acquire and reconstruct transmission CT data using our triple-camera research SPECT system. The transmission CT data are used to obtain attenuation maps of the object being scanned. These maps are used by the $3 D$ reconstruction algorithm to improve quantification of the SPECT images. 
3) To evaluate the accuracy of a surface-fitting program for registering 3D SPECT and positron ernission tomographic (PET) images. The ability to register or fuse $3 \mathrm{D}$ image data acquired from SPECT, PET, CT and MRI is required for the effective utilization of radiolabeled monoclonal antibodies (MoAbs) for diagnosis and therapy.

\section{SCIENTIFIC PERFORMANCE REPORT}

\section{A. UPGR.ADE OF TRIPLE-CAMERA SPECT SYSTEM}

A recently awarded University Research Instrumentation grant (DE-FG05-90ER75577) funded by the Department of Energy has enabled us to improve significantly the quantitative capability of our research SPECT system. We have sbtained new ultra-high resolution collimators, an improved imaging table, upgraded scintillation crystals, photomultiplier tubes, electronics, new acquisition and data processing software, and improved SPECT reconstruction software. This upgraded system has been of critical importance in assisting us in evaluating improved 3D SPECT' acquisition and reconstruction methods to extract quantitatively accurate measurements.

\section{B . QUANTITATIVE SPECT IMAGING: FILTEREd BACKPROJECTION AND ITERATIVE 3D RECONSTRUCTION ALGORITHMS FOR SPECT}

\section{B.1. EFfects of AtTenuation AND Detector Response}

Two major factors which degrade quantitative accuracy in SPECT imaging of the brain are attenuation and detector response. The purpose of this work was to implement two reconstruction methods which compensate for attenuation and detector response (a filtered backprojection method with Metz filter and Chang attenuation comperssation and a 3D maximum likelihood-expectation maximization method (ML-EM)) and compare the methods in terms of quantitative accuracy. The methods were tested on simulated data of the 3D Hoffman brain phantom. The simulation incorporated attenuation and distance-dependent detector response. The rms error was measured in the reconstructed images in the gray matter, white matter, and ventricle regions. The results in the white matter showed the 3D ML-EM method over a range of iteration stopping points had a smaller error than the filtered backprojection method over a similar range of power factors. In the gray matter the reverse was found, and this was attributed to an edge artifact in the 3D ML-EM images. Reconstruction times for the 3D ML-EM method have been greatly reduced through efficient coding, limited source support, and computing attenuation factors only along rays perpendicular to the detector.

\section{B.2. MaXmuM LIKELIHOOD-EXPECTATION MAXimization (ML-EM)}

A maximum likelihood-expectation maximization (ML-EM) reconstruction algorithm for SPECT which models non-uniform attenuation and distance-dependent detector response in 3D has been implemented and compared with the filtered backprojection method (FB) in terms of the signal-to-noise ratio (SNR) in the reconstructed image. The methods were tested on simulated 
projection data of an elliptical cylinder with uniform activity except for two spherical lesions with $50 \%$ decreased activity. The lesions were $2 \mathrm{~cm}$ in diameter and located on and $7 \mathrm{~cm}$ off the axis of rotation. An ensemble of 10 projection datasets were simulated incorporating the effects of 3D distance-dependent detector response, attenuation, and Poisson noise. The total counts per 0.36 $\mathrm{cm}$ thick slice were 075,000 . SNR was computed in the slice through the lesion center as the average lesion contrast divided by the standard deviation of lesion contrast over the 10 reconstructed images. Lesion contrast, defined as mean background counts minus mean lesion courts divided by mean background counts, was computed using a $1.5 \mathrm{~cm}$ diameter circular ROI at the known lesion location. The ML-EM algorithm was tested at 10, 25, 50 and 100 iterations. The FB method employed Chang attenuation compensation (one iteration) and Metz filter (powers 2,6 , and 12) for detector response compensation. For both lesion locations, the ML-EM method had better SNR than the FB rnethod for nearly all of the reconstruction parameters tested. For the off axis lesion, for example, the ML-EM SNR was 31.4 at 50 iterations compared with 19.6 and 15.7 for the FB with Metz filter powers of 2 and 6, respectively. Thus, a substantial improvement in SNR can be achieved with the 3D ML-EM method compared to FB.

\section{B.3. Bayesian RECONSTRLCTION Algorithm}

Statistical estimation procedures for reconstructing SPECT images are promising in part because they are based on a potentially accurate model of the probability of the projection data given the radiopharmaceutical distritution. Photon attenuation and scatter, crystal resolution, and spatially-varying collimator resolution are the primary physical factors affecting this probability, yet computational requirements for nodeling these effects can be prohibitive. We have developed computationally-efficient implementations of the Bayesian algorithms. In these implementations, a set of possibly-active voxels is initially identified, and photon-detection probabilities are precomputed and stored for these voxels only. Activities of other voxels are fixed at zero. Scatter effects are removed from the detection probabilities while treating properly the Poisson character of the projection data by introducing a set of complete data that distinguishes scattered and nonscattered ptotons. The detection probabilities used thus combine attenuation effects, crystal resolution, and spatially-varying collimator resolution. A single, pre-calculated attenuation probability is assumed for all photons emitted from a given voxel and registered at a given detector angle. Collimator and crystal resolution effects are pre-calculated and stored as a function of both the voxel-to-collimator distance and the location of the voxel's projection within the bin containing that projection. About 40 megabytes of mernory are required to reconstrsct the 3D Hoffman brain phantom at high resolution ( $3.6 \times 3.6 \times 3.6 \mathrm{~mm}$ voxels) from 120 angles of data with $128^{2}$ bins per angle. On a Sun SPARCstation II computer, this implementation reconstructs the brain phantom in five minutes per iteration, which is about eight seconds per iteration per slice.

\section{ACQuisition OF TRANSmission CT DATA WITH THREE-HEAded SPECT SYSTEM.}

\section{C.1. Use of a Specially Designed Curved Transmission Source and PARALlel-HOLE COLlimation}

Transmission computed tomography (TCT) methods have been used in single photon emission computed tomography (SPECT) to obtain the patient attenuation distribution for more 
accurate attenuation compensation. We have constructed a TCT system for a three-headed SPECT camera and have determined the accuracy of reconstructed attenuation coefficient values using experimental phantom data. A fillable slab source with a parallel hole collimator mounts between two camera heards and thus allows TCT data acquisition with the opposing head. The source $(50 \times 12 \times 1 \mathrm{~cm})$ is curved to provide greater patient clearance during rotation, and the collimatur is designed for high sensitivity (hole length $=1.2 \mathrm{~cm}$, diameter $=0.25 \mathrm{~cm}$ ). For this study the source was filled with $29 \mathrm{mCi}$ of Tc- $99 \mathrm{~m}$ solution. Detected scatter-to-primary ratios were measured with and without source collimator in a TCT projection of a water-filled eiliptical cylinder $(22 \times 30 \times 4$ $\mathrm{cm})$. Source collimation reduced the scatter-to-primary ratio from 0.24 to 0.05 . The accuracy of reconstructed TCT images was determined for a chest phantom consisting of two "lungs" (880 and $1175 \mathrm{ml})$ and a cylindrical teflon "spine" $(3.6 \mathrm{~cm}$ diameter $)$ in a water-filled elliptical cylinder. One lung contained only air, the other contained a homogeneous mixture of $0.4 \mathrm{~cm}$ diameter Styrofoam beads and water. The linear attenuation coefficient, $m$, for the latter lung was $0.057 / \mathrm{cm}$ and for the spine was 0.289 per $\mathrm{cm}$. The scan time was one hour, and the slice thickness was $7 \mathrm{~cm}$ to reduce noise. With source collimation the reconstructed values were within $2 \%$ of the true $\mathrm{m}$ in all regions of the phantom. Without source collimation reconstructed values underestimated the true $m$ in all regions by approximately $12 \%$, and this can be attributed to the detection of scattered photons. Thus, proper source collimation can substantially improve the quantitative accuracy of reconstructed TCT images.

\section{C.2 Use of a line Source and a Specially Designed Slit Source SOURCE COLLIMATOR FOR FAN BEAM TCT: INITIAL STUDY}

The objective of this work was to determine the feasibility of using a line source/slit collimator (LS/SC) assembly coupled with an appropriate fan beam collimator with our triplecamera SPECT system to obtain transmission computed tomographic (TCT) data for brain and body attenuation maps. These maps may be useful for the following reasons: 1)To improve SPECT' quantification by providing accurate detection probabilities for our quantitative reconstruction algorithms; and 2)To provide anatomical data that would facilitate the registration of serial SPECT studies, or the registration of 3D SPECT image data sets with 3D image data sets from volumetric positron imaging (VPI), $x$-ray CT, and magnetic resonance imaging (MRI). The slit collimator minimizes additional radiation dose to the patient, and it also minimizes additional scatter in the transmission data. The $20 \mathrm{~cm}$ long line source ( $1 \mathrm{~mm}$ capillary tube) was filled with approximately $5 \mathrm{mCi}$ of Tc- $99 \mathrm{~m}$ solution and placed directly on top of the $20 \mathrm{~cm}$ long slit collimator.

For this initial study, the complete LS/SC assembly was positioned directly above an uncollimated scintillation camera. The line source was placed parallel to the axis-of-rotation and perpendicular to a line intersecting the center of one of the scintillation crystals of our triple-camera SPECT system. Thus, the LS/SC assembly was positioned between the other two cameras, since the three are equally spaced at 120 degree intervals.

The use of an uncollimated scintillation camera allowed us to position the source at several different distances above the detector; hence, we were able to vary the "focal length" of the fan beam transmission source to determine the useful field-of-views (FOV) and the magnitude of truncation artifacts on the reconstructed TCT maps. Of course, a practical configuration would require that a fan beam collimation be placed on the TCT scintillation camera, particularly if one wanted to simultaneously acquire SPECT data with the remaining two cameras of the triple-camera 
system. Although the FOV generally increases as the LS/SC is positioned further from the camera, the maximum FOV possible is limited at distances greater than approximately $110 \mathrm{~cm}$ because of mechanical constraints. Beyond $110 \mathrm{~cm}$ the diverging fan of gamma rays impinges on the housings of the other two SPECT cameras that are located at plus and minus 120 degrees from the I'CT camera, particularly if these cameras are positioned as they would be to acquire SPECT projection data. It was determined that a distance of between 40 and $50 \mathrm{~cm}$ would be appropriate for imaging the patient's head.

For body imaging, a distance of about $90 \mathrm{~cm}$ results in a useful FOV that is about $30 \mathrm{~cm}$. This FOV is not quite adequate to obtain non-truncated TCT data for many subjects. To investigate the effectiveness of the LS/SC assembly, TCT scans were obtained of an elliptical cylinder $(31 \mathrm{~cm}$ by $22 \mathrm{~cm}$ ) containing anatomically shaped lung inserts for several different LS/SC locations above the TCT scintillation camera. The attenuation maps obtained with the LS/SC positioned at the 90 $\mathrm{cm}$ demonstrated only minimal artifacts. The artifacts were confined mainly near the edges of the elliptical cylinder along the major axis.

We, and other groups, are currently investigating the significance of TCT truncation as it relates to SPECT attenuation compensation. It is reasonable to expect thai minor truncation will not markedly degrade the SPECT reconstruction, and should provide improved quantification as compared, for example of using an assumed constant attenuation coefficient. Our initial results indicate that TCT will be useful in improving SPECT quantification, and may be useful for providing additional anatomical information that could facilitate image registration for some SPECT studies.

\section{IMAGE Registration and Fusion: Evaluation of ACCuRACy Of Surface- FIT METHOD}

The purpose of this study was to measure the accuracy of a surface fitting image registration technique when applied to positron emission tomography (PET) and magnetic resonance (MR) images of human brains and to investigate automated, operator-independent means of surface detection.

Five multi-slice PET studies (two [0-15] $\mathrm{H}_{2} \mathrm{O}$ and three [F-18] FDG) were performed on five volunteers as well as T1-and T2-weighted volume MR scans. Brain contours were defined for each PET slice by an automated technique using a first derivative maximum as the edge criterion. MR brain edges were determined with a threshold set at approximately one-half the average pixel level inside the brain. Both PET and MR contours were smoothed to improve fitting. Surfaces were defined from each of the multi-slice brain edges, and transformation pararneters were determined via a non-linear least-squares fit for rotating and translating the PET surfaces to match ihe MR surfaces. External markers placed on the subjects' forehead and temple regions were used to measure the accuracy of the surface fits by comparison of the marker positions in the MR scans to the PET scan positions after transformation vir the surface fit parameters. The marker positions were determined by calculating centroids in the original image sets.

The PET and MR brain surfaces fit well, with scaling of less than $1 \%$ required for the best surface fit. Errors in registration were determined to be less than $1.5 \mathrm{~mm}$ in the transverse planes and less than $3 \mathrm{~mm}$ in the axial direction in each of the 5 studies. These errors include the increased effect of angular misregistration for points outside the head. 
This technique of edge determination resulted in surfaces which matched well without scaling, and marker errors represent registration accuracy sufficient for most registration goals.

\section{RELATED RESEARCH SUPPORTED IN PART BY DOE GRANT DE- FG05-89ER6089d}

This DOE rescarch project benefits greatly as a result of synergism with other related research projects at Duke dirtcted towards the development of radiolabeled MoAbs and improved quantitative methodology for SPECT. The complementary nature of these related projects facilitates progress in this DOE project. For exampie, Professor Jaszczak directs a research project (CA33541) funded by the National Cancer Institute (NCI) to improve SPECT imaging and lesion detection using the widely available nuclide of Technetium ( $99 \mathrm{~m} \mathrm{Tc}$ ). The projection involves the development of reconstruction algorithms for converging beam SPECT and improving Monte Carlo modeling of the SPECT acquisition process. The scientific knowledge, algorithms, and programs resuiting from that project can be effectively utilized by this DOE project.

Our colleague, Professor Valen Johnson, in the Institute of Statistics and Decision Sciences, directs a project directed toward the development of appropriate statistical reconstruction algorithms for PET and SPECT. In this DOE project, we intend to develop appropriate physical models of the system response functions for the ciinically relevant nuclides ${ }^{131} \mathrm{I},{ }^{123} \mathrm{I},{ }^{11} \mathrm{I}$ In, and ${ }^{211}$ At. These physical models will then be integrated with the statistical Bayesian models being developed by Professor Johnson.

Similarly, our colleague, Professor Michael Zalutsky, directs a research project (CA42324) funded by NCI to develop improved methods for labeling MoAbs with ${ }^{131}{ }^{I}$ and ${ }^{211}$ At. The scientific knowledge and methodology resulting from our DOE proposal will facilitate progress in Dr. Zalutsky's project.

Dr. Zalutsky also directs a research project (DE-FG05-89-ER60789) funded by DOE to develop methods to label MoAbs using nuclides of iodine $\left({ }^{124} \mathrm{I}\right)$ and fluorine $\left({ }^{18} \mathrm{~F}\right)$. We intend to utilize the results from that research project to compare the advantages and disadvantages of SPECT and PET to quantify biodistributions of 123 I- and ${ }^{124}$ I-labeled MoAbs, respectively.

The synergistic and complementary nature of Duke research projerts relating to SPECT, PET, and labeled MoAbs offers significant advantages tc this DOE proposa'.

Summaries of scientific findings in these related areas can be found in the publications listed below.

V. PUBLICATIONS FOR 1991-1992

A. Journal articles, Chapters, and Published proceedings

1. Gilland DR, Jaszczak RJ, Greer KL, Cnleman RE: Quantitative SPECT reconstruction of Iotine-123 Data. J Nucl Med 32:527-533, 1991. 
2. Gilland DR, Jaszczak RJ, Turkington TG, Greer KL, Coleman RE: Quantitative SPECT imaging with Indium-111. IEEE, Trans Nucl Sci 38:761-766, 1991.

3. Jaszczak RJ, Hoffman DC: Variance propagation for SPECT with energy-weighted acquisition. IEEE Trans Nucl Sci 38:739-747, 1991.

4. Jaszczak RJ, Li J, Wang H, Greer $\mathrm{KL}$, Coleman RE: Three dimensional reconstruction of combined cone beam and parallel beam data. Phys Med Biol, in press, 1992.

5. Gilland DR, Tsui BW, Metz CE, Jaszczak RJ, Perry R: An evaluation of maximum likelihood EM recoinstruction by ROC analysis: J Nucl Med, in press, 1992.

6. Gilland DR, Jaszczak RJ, Liang Z, Greer KL, Coleman RE: Quantitative SPECT brain imaging: Effects of attenuation and collimator response. IEEE Trans Nucl Sci, submitted, 1992 .

7. Turkington TG, Jaszczak RJ, Greer KL, Coleman RE: Correlation of SPECT images of a three-dimensional brain phantom using a surface fitting technique. IEEE Trans Nucl Sci, submitted, 1992.

8. Gilland DR, Jaszczak RJ, Greer KL, Coleman RE: SPECT quality control: Generation and reduction of artifacts. In: Proceedings of the SE Chapter of the Society of Nuclear Medicine, in press, 1992.

9. Gilland DR, Jaszczak RJ, Liang Z, Greer KL, Coleman RE: Quantitative SPECT brain imaging: Effects of attenuation and collimator response. In: Conference Record of the 1991 IEEE Nuclear Symposium (Santa Fe, NM, 1991) in press.

10. Turkington TG, Jaszczak RJ, Greer KL, Coleman RE: Correlation of SPECT images of three-dimensional brain phantom using a surface fitting technique. In: Conference Record of the 1990 IEEE Nuclear Symposium (Santa Fe, NM, 1991) in press.

11. Jaszczak RJ, Tsui BMW: Single photon emission computed tomography. In: Principles of Nuclear Medicine, eds. HN Wagner and Z Szabo (W.B. Saunders, Philadelphia, 1993), in preparation.

12. Jaszczak RJ, Hoffman EJ: Scatcer and attenuation. In: Principles of Nuclear Medicine, eds. HN Wagner and Z Szabo (W.B. Saunders, Philadelphia, 1993), in preparation.

\section{B. ABSTRACTS}

13. Turkington TG, Jaszczak RJ, Gilland DR, Greer KL, Coleman RE: Quantitation of Indiurn111 activity in SPECT. J Nucl Med 32(5):986 (Abstract), 1991.

14. Gilland DR, Jaszczak RJ, Coleman RE: Effects of non-uniform attenuation compensation in SPECT using acquired transmission data. J Nucl Med 32(5): 1067, (Abstract), 1991. 
15. Turkington TG, Jaszczak RJ, Pelizzari CA, Harris CC, MacFall JR, Hoffman JM: Measurement of accuracy in registration of PET and SPECT brain images to MR images. Radiology 181(P): 186, (Abstract), 1991.

16. Gilland DR, Jaszczak RJ, Turkington TG, Greer KL, Coleman RE: Transmission data acquisition with a three headed SPECT system. Society of Nuclear Medicine 39th Annual Meeting, Los Angeles(Abstract), June 1992, accepted.

17. Gilland DR, Bowsher JE, Jaszczak RJ, Coleman RE: Improved signal-to-noise with 3D MLEM reconstruction. Society of Nuclear Medicine 39th Annual Meeting, Los Angeles(Abstract), June 1992, accepted.

18. Turkington TG, Jaszczak RJ, Hoffman JM, MacFall JR, Harris CC, Kilts CD, Pelizzari CA, Coleman RE: Accuracy of surface fit registration of PET and MR brain images. Society of Nuclear Medicine 39th Annual Meeting, Los Angeles(Abstract), June 1992, accepted.

19. Bowsher JE, Gilland DR, Floyd CE, Jaszczak RJ, Johnson VE, Coleman RE: Threedimensional iterative reconstruction for SPECT. Society of Nuclear Medicine 39th Annual Meeting, Los Angeles(Abstract), June 1992.

20. Jaszczak RJ, Li J, Wang H, Coleman RE: SPECT collimation having spatially variant focusing (SVF). Society of Nuclear Medicine 39th Annual Meeting, Los Angeles (Abstract), June 1992, accepted.

C. Related publications supported IN PART By DOE GRANT DE-FG05. 91ER60894

21. Koral KF, Wang X, Zasadny KR, Clinthorne NH, Rogers WL, Floyd CE, Jaszczak RJ: Testing of local gamma-ray scatter fractions determined by spectral fitting. Phys Med Biol $36: 177-190,1991$.

22. Liang Z, Jaszczak RJ, Coleman RE, Johnson V: Simultaneous reconstruction, segmentation, and edge enhancement of piecewise continuous images with intensity-level information. Med Phys 18:394-401, 1991.

23. Liang $Z$, Jaszczak $R$, Coleman $R$ : On reconstruction and segmentation of piecewise continuous images. In: Information Processing in Medical Imaging, 12th International Conference (Springer-Verlag, Berlin, 1991), pp. 94-104.

24. Smith MF, Floyd CE, Jaszczak R ${ }^{\mathrm{T}}$, Coleman RE: Reconstruction of SPECT images using generalized matrix inverses. IEEE Trans Med Imaging, submitted, 1992.

25. Smith M, Floyd C, Jaszczak R, Coleman R: Three dimensional photon detection kernels and their application to SPECT reconstruction. Phys Med Biol, in press, 1992.

26. Liang Z, Jaszczak RJ, Turkington 'TG, Gilland DR, Coleman RE: Simultaneous compensation for attenuation, scatter, and detector response of SPECT reconsiruction in three dimensions. Phys Med Biol, in press, 1992. 
27. Warig $\mathrm{H}$, Jaszczak RJ, Coleman RE: Solid geometry based object model for Monte Carlo simulated emission and transmission tomographic imaging systems. IEEE Trans Med Imaging, in press, 1992.

28. Li J, Jaszczak RJ, Greer KL, Coleman RE: Experimental evaluation of combined cone beam with parallel hole collimation SPECT data. In: Conference Record of the 1991 IEEE Nuclear Science Symposium (Santa Fe, NM 1991), in press.

29. Liang Z, Jaszczak R, Coleman R: An efficient 3D unified projector-backprojector for SPECT reconstruction. In: Conference Record of the 1991 IEEE Nuclear Science Symposium. (Santa Fe, NM 1991), in press.

30. Wang H, Jaszczak RJ, Gilland DR, Greer KL, Coleman RE: Solid geometry based modelling of non-uniform atienuation and Compton scattering in objects for SPECT imaging systems. In: Conference Record of the 1991 IEEE Nuclear Science Symposium (Santa Fe, NM. 1991), in press.

31. Smith MF, Floyd CE, Jaszczak RJ, Coleman RE: Evaluation of projection pixel-dependent and pixel-independent scatter correction in SPECT. In: Conference Record of the 1991 IEEE Nuclear Sciense Symposium (Santa Fe, NM 1991), in press.

32. Li J, Jaszczak RJ, Greer KL, Coleman RE: Experimental evaluation of combined cone beam with parallel hole collimation SPECT data. IEEE Trans Nucl Sci, submitted, 1992.

33. Liang Z, Jaszczak R, Coleman R: An efficient 3D unified projector-backprojector for SPECT reconstruction. IEEE Trans Nucl Sci, submitted, 1992.

34. Smith MF, Floyd CE, Jaszczak RJ, Coleman RE: Evaluation of projection pixel-dependent and pixel-independent scatter correction in SPECT. IEEE Trans Nucl Sci, submitted, 1992.

35. Wang H, Jaszczak RJ, Gilland DR, Greer KL, Coleman RE: Solid geometry based modelling of non-uniform attenuation and Compton scattering in objects for SPECT imaging systerns. IEEE Trans Nucl Sci, submitted, 1992.

36. Leichner PK, Koral KF, Jaszczak RJ, Green AJ, Chen G, Roeske JC: Imaging techniques and treatment planning in radioimmunotherapy (RIT): Med Phys, submitted, 1992.

37. Schold SC, Zalutsky MR, Glantz MJ, Coleman RE, Friedman AH, Jaszczak RJ, Bigner SH, Bigner DD: Disuribution and dosirnetry of 1231 labeled monoclonal antibody $81 \mathrm{C} 6$ in patients with anaplastic glioma. Invest Radiology, submitted, 1992.

\section{ABSTRACTS SUPPORTED IN PART BY DOE GRANT DE-FG05-91ER60894}

38. Smith MF, Floyd CE, Jaszczak RJ, Coleman RE: Reconstructing SPECT images using three-dimensional photon detection kernels. J Nucl Med 35(5):1068 (Abstract), i991.

39. Liang Z, Jaszczak R, Coleman R: A 3D model for simultaneous compensation of non-uniform attenuation and collimation divergence of SPECT image reconstruction. J Nucl Med 32(5): 
917(Abstract), 1991.

40. Wang H, Jaszczak RJ, Coleman RE: Monte Carlo model of non-uniform attenuating phantoms with irregular-shaped regions for SPECT imaging systems. J Nucl Med 32(5):1065, (Abstract), 1991.

41. Li J, Jaszczak RJ, Wang H, Greer KL, Coleman R: Evaluation of combined cone beam with parallel hole collimation SPECT data. J Nucl Med 32(5):956, (Abstract), 1991.

42. Liang $\mathrm{Z}$, Coleman RE: Restoration for detector response in high resolution PET image reconstruction. Society of Nuclear Medicine 39th Annual Meeting (Abstract), Los Angeles, June, 1992, accepted.

43. Li J, Wang H, Jaszczak RJ, Coleman RE: A cone beam reconstruction algorithm with a displaced center-of-rotation. Society of Nuclear Medicine 39th Annual Meeting, Los Angeles (Abstract), June 1992, accepted.

44. Zeng GL, Gullberg GT, Jaszczak RJ, Li J: Fan beam convolution reconstruction algorithms for variable focal length collimators. Society of Nuclear Medicine 39th Annual Meeting, Los Angeles (Abstract), June 1992, accepted.

45. Koral KF, Luo J, Wang X, Floyd CE, Jaszczak RJ, Ljungberg M: Tc-99m Compton-scatter correction: Dual-energy-window multiplier from Monte Curlo simulation. Society of Nuclear Medicine 39th Annual Meeting, Los Angeles (Abstract), June 1992, accepted.

E. Related MoAb Publications Supported by DR. Zalutsky's DOE grant (DE-FG05-89ER60789)

46. Garg S, Garg PK, Zalutsky MR: N-succinimidyl 5-(trialkylstannyl)-3-pyridinecarboxylates: A new class of reagents for protein radioiodination. Bioconjugate Chem 2:50-56, 1991.

47. Garg PK, Garg S, Zalutsky MR: Fluorine-18 labeling of monoclonal antibodies and fragments with preservation of immunoreactivity. Bioconjugate Chem 2:50-56, 1991.

48. Garg S, Garg PK, Zalutsky MR: N-succinimidyl 5-(trialkylstannyl)-3-pyridinecarboxylates: A new class of reagents for protein radioiodination. Bioconjugate Chem 2:50-56, 1991.

49. Garg PK, Garg S, DeGraff WG, Zalutsky MR, Mitchell JB: 4-Fluorobenzylamine and phenylalanine methyl ester conjugates of 2-nitroimidazole: evaluation as hypoxic cell radiosensitizers. Int J Radiat Oncol Biol Phys, in press, 1992.

50. Vaidyanathan G, Zalutsky MR: Labeling proteins with fluorine-18 using N-succinimidyl 4[18F] fluorobenzoate. Nucl Med Biol, in press, 1992.

51. Zalutsky MR, Garg PK, Vaidyanathan G, Garg S: Methods for the radiohalogenation of antibodies. In: Applications for Enzyme Biotechnology, Plenum Press, in press, 1992.

52. Zalutsky MR, Garg PK, Johnson SH, Coleman RE: Fluorine-18 antimyosin monocional antibody fragments: Preliminary investigation in a canine myocardial infarct model. J Nucl 
Med, submitted, 1992.

53. Garg S, Garg PK, Bigner DD, Zalutsky MR: Selective localization of an 18F labeled Mel-14 monoclonal antibody $\left.\mathrm{F}(\mathrm{ab})_{2}\right)_{2}$ fragment in a subcutaneous human glioma xenograft moxlel, $\mathrm{J}$ Nucl Med, submitted, 1992.

54. Schuster JM, Dewhirst MW, Bigner DD, Zalutsky MR: Distribution of radiolabeled monoclonal antibody $F\left(a b^{\prime}\right)_{2}$ fragments in a subcutaneous xenograft model following localized hyperthermia: Temperature effects, Int. J Radiat Oncol Biol Phys, submitted, 1992.

55. Vaidyanathan G, Bigner DD, Zalutsky MR: Fluorine-18 labeled monoclonal antibody fragments: A potential approach for combining radioimmunoscintigraphy and positron emission tomography, Cancer Res, submitted, 1992.

F. Selected Invited Presentations (1991.1992)

56. Jaszczak RJ: Overview of SPECT: Instrumentation and image reconstruction. Canadian National Nuclear Medicine Meeting (Banff, Alberta, Canada, April 9-12, 1991).

57. Gilland DR, Jaszczak RJ, Greer KL, Coleman RE: SPECT quality control: Generation and reduction of artifacts. SE Chapter of the Society of Nuclear Medicine (Miami Beach, October, 1991).

58. Jaszczak RJ, Gilland DR: SPECT simulations of the Hoffman JD brain phantom. SPECT Monte Carlo Workshop (Worcester, Mass., October 25-27, 1991).

59. Wang H, Jaszczak RJ: SPECT Monte Carlo using constructive solid geometry modeling. SPECT Monte Carlo Workshop (Worcester, Mass., October 25-27, 1991).

60. Jaszczak RJ: Acquisition factors that affect SPECT quantification. Australian and New Zealand Society of Nuclear Medicine (Adelaide, Australia, May 4, 1992).

61. Jaszczak RJ: The factors that affect SPECT reconstruction. Australian and New Zealand Society of Nuclear Medicine (Adelaide, May 5, 1992).

62. Jaszczak RJ: Iterative reconstruction methods. Australian and New Zealand Society of. Nuclear Medicine (Adelaide, May 3, 1992).

63. Jaszczak RJ: Review of scatter correction methods. Australian and New Zealand Society of Nuclear Medicine (Adelaide, May 3, 1992). 

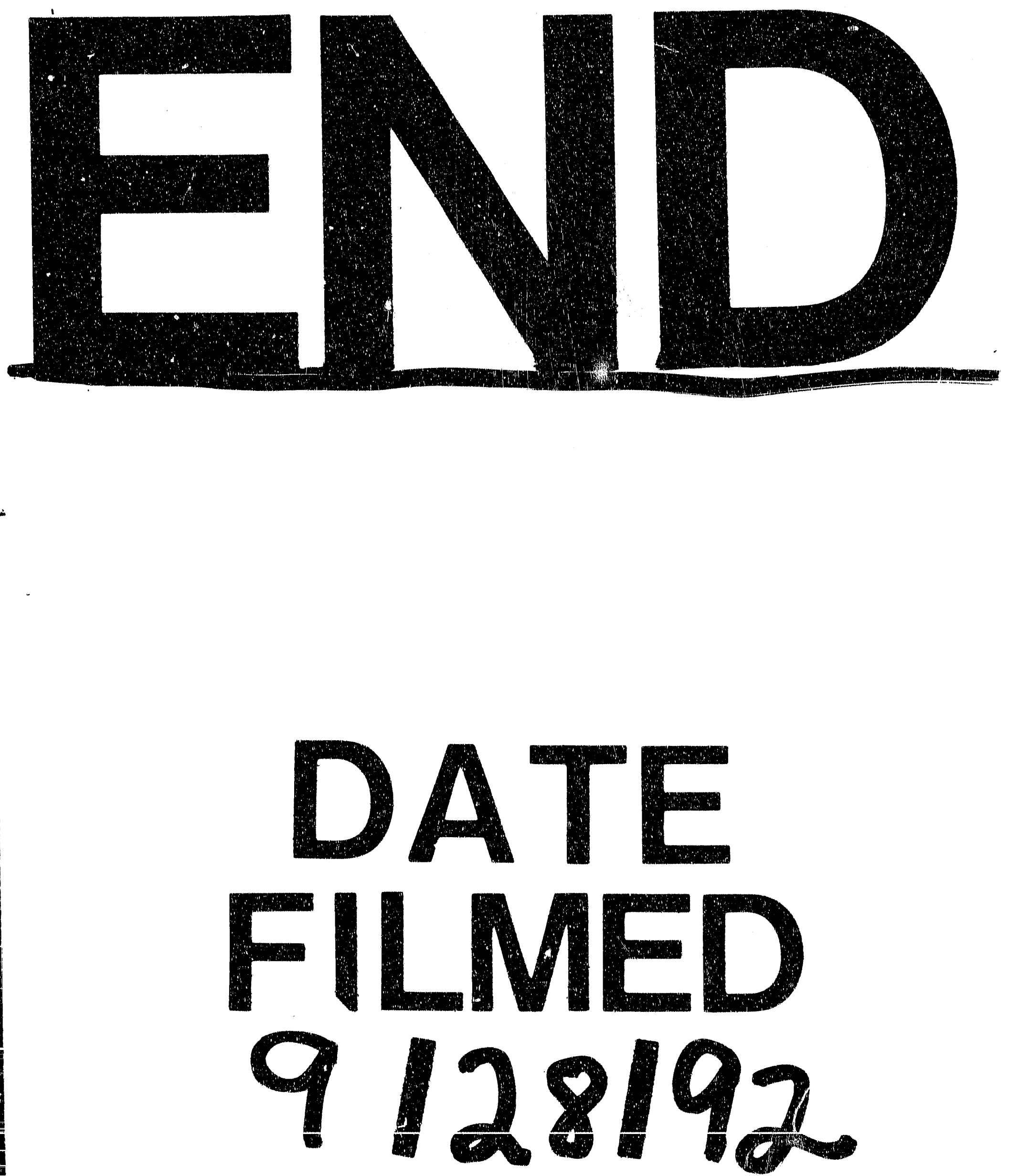
